\title{
L-Deprenyl exerts cytotoxicity towards acute myeloid leukemia through inhibition of mitochondrial respiration
}

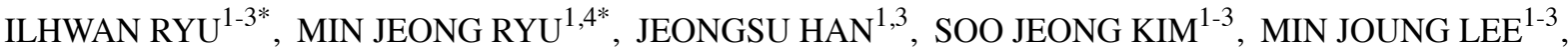 \\ XIANSHU JU ${ }^{2,3}$, BYEONG HYEON YOO ${ }^{1-3}$, YU LIM LEE ${ }^{1-3}$, YUNSEON JANG ${ }^{1-3}$, IK-CHAN SONG ${ }^{5}$, \\ WOOSUK CHUNG ${ }^{2,6,7}$, EUNGSEOK OH ${ }^{8,9}$, JUN YOUNG HEO ${ }^{1-3,10}$ and GI RYANG KWEON ${ }^{1,2,4}$

\begin{abstract}
Departments of ${ }^{1}$ Biochemistry and ${ }^{2}$ Medical Science, ${ }^{3}$ Infection Control Convergence Research Center, ${ }^{4}$ Research Institute for Medical Science, Chungnam National University School of Medicine; ${ }^{5}$ Department of Chungnam National University School of Medicine; Departments of ${ }^{7}$ Anesthesiology and Pain Medicine, and ${ }^{8}$ Neurology, Chungnam National University Hospital; ${ }^{9}$ Department of Neurology, ${ }^{10}$ Brain Research Institute, Chungnam National University School of Medicine, Daejeon 35015, Republic of Korea
\end{abstract} \\ Internal Medicine, Chungnam National University Hospital; ${ }^{6}$ Department of Anesthesiology and Pain Medicine,
}

Received March 15, 2018; Accepted September 17, 2018

DOI: $10.3892 /$ or.2018.6753

\begin{abstract}
The identification of large numbers of genetic mutations in immature myeloid cells has made it difficult to identify specific targets for acute myeloid leukemia (AML) therapy. Although current pharmacological targets for controlling cancer are focused on identifying genetic mutations, it is hard to develop the specific drugs to achieve complete remission due to complex and variable genetic mutations. To overcome the failure of the genetic mutation theory, the present study targeted mitochondrial metabolism as a strategy for inducing anti-leukemic activity, based on evidence that AML cells have an abnormally high amount of mitochondria and that somatic mutations can alter metabolic flux in cancer. It was found that L-deprenyl, which is clinically available for the treatment of Parkinson's disease, exerts anti-mitochondria activity in KG-1 $\alpha$ cells, as assessed by detection of oxygen consumption rate (OCR) and extracellular acidification (ECAR) using XF analyzer, respectively. Using a luciferase assay for detecting adenosine triphosphate (ATP) content, it was found that suppression of mitochondrial activity led to ATP depletion and was associated with potent
\end{abstract}

Correspondence to: Professor Jun Young Heo or Professor Gi-Ryang Kweon, Department of Biochemistry, Chungnam National University School of Medicine, 266 Munhwa-ro, Daejeon 35015, Republic of Korea

E-mail: junyoung3@gmail.com

E-mail: mitochondria@cnu.ac.kr

*Contributed equally

Key words: monoamine oxidase-B, acute myeloid leukemia, mitochondrial respiration, adenosine triphosphate, anticancer activity cytotoxic activity. L-Deprenyl is known to target monoamine oxidase-B (MAO-B) on the outer membrane of mitochondria, therefore, the activity of MAO-A and -B was measured based on the fluorometric detection of $\mathrm{H}_{2} \mathrm{O}_{2}$ produced by the enzyme reaction. Notably, MAO-A and -B activity was low in AML cells and the present findings suggested that the anticancer effect of L-deprenyl was independent of MAO-B. Change of mitochondrial respiration- and glycolysis-related gene expression levels were measured by reverse transcription-quantitative polymerase chain reaction. Consistent with the aforementioned results, treatment with L-deprenyl reduced the mRNA level of mitochondrial respiration- and glycolysis-related genes. Collectively, the present results identify L-deprenyl as a novel candidate for the treatment of AML through inhibition of mitochondrial respiration.

\section{Introduction}

Acute myelogenous leukemia (AML) is a hematopoietic cancer with a high mortality rate, and $>50 \%$ of patients with AML show no response to chemotherapy (1). Although individual therapeutic modalities have been developed and applied to control AML, the diversity of gene mutations and heterogeneity among cancer cells has largely confounded efforts to achieve complete remission $(2,3)$. To overcome difficulties in identifying effective drug targets, the focus in AML research has increasingly turned from gene regulation to organelle-based therapy (4).

Mutant forms of the tyrosine kinase FMS-like tyrosine kinase 3-Internal tandem duplication (FLT3-ITD), encoded by an AML-associated gene, induce reactive oxygen species (ROS) production and DNA damage, sequentially contributing to genetic mutations and a poor prognosis (5). Mitochondria are responsible for producing $~ 90 \%$ of intracellular ROS, and persistent ROS production is associated with AML development (6). NADPH oxidase-2-mediated superoxide production stimulates mitochondrial transfer from 
bone marrow stromal cells to leukemic blast cells through AML-derived tunneling nanotubes. Intact mitochondrial transfer causes higher oxidative phosphorylation activity, which mediates the supplementation of biosynthetic precursors and adenosine triphosphate (ATP), which finally induces rapid proliferation of AML cells (7). In addition, depletion of autophagy results in failure to remove dysfunctional mitochondria and causes dysregulation of the glycolytic pathway, thereby reducing the survival rate of patients with AML (8). Thus, regulation of mitochondrial-related metabolic flow has potential as a treatment strategy against AML or other cancer types characterized by somatic mutations that affect mitochondrial metabolism.

L-Deprenyl is a highly selective and irreversible inhibitor of monoamine oxidase B (MAO-B) (9) that prevents the deamination of dopamine, thus maintaining the dopamine concentrations in the synapses of the nigrostriatal pathway (10). This dopamine concentration-maintaining action of L-deprenyl is the basis for its use in the therapy of Parkinson's disease. As ROS are generated through deamination, L-deprenyl also acts to decrease ROS levels in neuronal cells, which alleviates the amount of dopaminergic neuronal cell death from exo- and endogenous insults (11). The high concentration of L-deprenyl is known to exert anticancer effects, exhibiting efficacy in reducing breast tumor size and decreasing tumor cell viability. There is also evidence that L-deprenyl decreases the activity of an estrogen-receptor (ER)-dependent intracellular signaling pathway in ER-positive human breast cancer (12). In addition to its effects on solid tumors, L-deprenyl also reduces the number of monoblastic leukemia cells by increasing production of norepinephrine, interferon- $\gamma$, cluster of differentiation $8^{+}$lymphocytes and natural killer cells in the spleen (13). However, whether an intracellular organelle-based mechanism underlies the antitumor effect of L-deprenyl, and whether this action is dependent on MAO-B inhibition, remains uncertain. Moreover, the involvement of changes in mitochondrial respiration in L-deprenyl-induced cytotoxicity has not been demonstrated. The present study investigated mitochondrial respiration as a novel target of L-deprenyl in AML cells, which undergo apoptotic cell death in response to L-deprenyl.

\section{Materials and methods}

Animals. Male FLT3-ITD knock-in and wild-type (WT) mice with a C57BL/6 background were purchased from Jackson Laboratory (Bar Habor, ME, USA). All animal experiments were conducted in the animal facility according to institutional guidelines (standard operating procedure), and approved by the Institutional Animal Care and Use Committee of Chungnam National University Hospital (CNUH-015-A0007-2). A total of 6 mice (aged 8 weeks, weighed $20 \pm 2 \mathrm{~g}$ ) were maintained in a controlled environment (12-h light/dark cycle; temperature, $22^{\circ} \mathrm{C} ; 55 \%$ humidity), and provided with food (cat. no. AIN-76A; Research Diets Inc., New Brunswick, NJ, USA) and water ad libitum.

Isolation of mouse bone marrow mononuclear cells (BMMNCs). Firstly, the mice were placed into a 1.5-liter volume plastic chamber, then exposed to $100 \% \mathrm{CO}_{2}$ at a flow rate of $0.25 \mathrm{l} / \mathrm{min}$. After the euthanasia, each end of the femur and tibia were cut off. Warm phosphate-buffered saline (PBS; $20 \mathrm{mM}$, pH 7.4) was then injected into the marrow cavity. Fluids were collected in 50-ml conical tubes containing $20 \mathrm{ml}$ Lymphoprep (Takeda Pharmaceuticals International GmbH, Zurich, Switzerland), which is a density gradient medium recommended for the isolation of MNCs. Centrifugation was performed at $800 \mathrm{x} \mathrm{g}$ for $20 \mathrm{~min}$ at $20^{\circ} \mathrm{C}$. As granulocytes and erythrocytes have a higher density than MNCs at the osmotic pressure of Lymphoprep, during the centrifugation process, granulocytes and erythrocytes sediment through the Lymphoprep layer and the MNCs with lower densities remain at the plasma Lymphoprep interface, which allows isolation of the BMMNCs. Subsequent to centrifugation, the BMMNC layer was collected using a Pasteur pipette, then washed with PBS and re-suspended in Dulbecco's modified Eagle's medium (DMEM; Thermo Fisher Scientific, Inc., Waltham, MA, USA) supplemented with $20 \%$ fetal bovine serum (GE Healthcare Life Sciences, Logan, UT, USA) and penicillin-streptomycin (Thermo Fisher Scientific, Inc.).

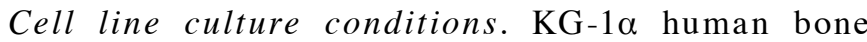
marrow-derived AML cells were obtained from the American Type Culture Collection (Manassas, VA, USA). KG-1 $\alpha$ cells were maintained in Iscove's modified Dulbecco's medium (Thermo-Fisher Scientific, Inc.) supplemented with $20 \%$ fetal bovine serum (GE Healthcare Life Sciences) and penicillin-streptomycin (Thermo Fisher Scientific Inc.) at $37^{\circ} \mathrm{C}$ in a humidified $5 \% \mathrm{CO}_{2}$ environment. HL-60 human blood-derived acute promyelocytic leukemia cells were obtained from the Korean Cell Line Bank (Seoul, South Korea). HL-60 cells were maintained in Roswell Park Memorial Institute medium (Welgene Inc., Gyeongsan, South Korea) supplemented with $10 \%$ fetal bovine serum (GE Healthcare Life Sciences) and penicillin-streptomycin (Thermo Fisher Scientific, Inc.) at $37^{\circ} \mathrm{C}$ in a humidified $5 \% \mathrm{CO}_{2}$ environment.

Cell viability assay. Cell viability was determined using Cell Counting Kit-8 (CCK-8; Dojindo Molecular Technologies, Inc., MD, USA). For the 24-h experiment, BMMNCs and $\mathrm{KG}-1 \alpha$ cells were plated at $1 \times 10^{4}$ per well in 96-well tissue culture plates and incubated at $37^{\circ} \mathrm{C}$ overnight. Following L-deprenyl (0.5-4 mM; cat. no. M003; Sigma-Aldrich; Merck KGaA, Darmstadt, Germany) and PBS (control) treatment for $24 \mathrm{~h}, \mathrm{CCK}-8$ reagents, which allow sensitive colorimetric determination of the number of viable cells, were added to each well. In acute experiments, i.e., treatment with L-deprenyl for $20 \mathrm{~min}$, once KG-1 $\alpha$ cells had been plated at $1 \times 10^{4}$ per well in 96-well tissue culture plates and incubated at $37^{\circ} \mathrm{C}$ for overnight, $\mathrm{CCK}-8$ reagents were added to the KG-1 $\alpha$ cells. After 40 min, L-deprenyl and PBS was added and the plate was incubated for an additional $20 \mathrm{~min}$. WST-8 is reduced by dehydrogenase in cells to form an orange-colored formazan. The amount of formazan and the number of living cells are in direct proportion. Absorbance was measured at a wavelength of $450 \mathrm{~nm}$ using a MultiSkan Ascent microplate spectrophotometer (Thermo Fisher Scientific, Inc.).

Measurements of MAO activity. MAO activity of the whole brain tissues of WT mice, the bone marrow cells of FLT3-ITD knock-in mice, and KG-1 $\alpha$ and HL-60 cells was determined using a commercially available kit (Amplex Red Monoamine 
Oxidase Assay kit; cat. no. A12214; Thermo Fisher Scientific, Inc.), following the manufacturer's protocols. Following sacrifice and removal of the brain, whole brain tissue was collected and then homogenized using a pellet pestle (cat. no. Z359971; Sigma-Aldrich; Merck KGaA) with radioimmunoprecipitation assay lysis (RIPA) buffer containing $10 \%$ phosphatase and protease inhibitor cocktail (Roche Diagnostics, Basel, Switzerland), and centrifuged at $14,000 \mathrm{x}$ g for $15 \mathrm{~min}$ at $4^{\circ} \mathrm{C}$. Bone marrow cells were collected with $20 \mathrm{mM}$ ice cold PBS ( $\mathrm{pH}$ 7.4) from the femur and tibia of FLT3-ITD knock-in mice. The bone marrow cells, KG-1 $\alpha$ cells and HL-60 cells were centrifuged at $14,000 \mathrm{x}$ g for $5 \mathrm{~min}$ at $4^{\circ} \mathrm{C}$ and then pellets were placed in RIPA buffer containing $10 \%$ phosphatase and protease inhibitor cocktail (Roche Diagnostics) and centrifuged at $14,000 \mathrm{x} \mathrm{g}$ for $15 \mathrm{~min}$ at $4^{\circ} \mathrm{C}$. Protein concentration in the supernatant was determined using a Bradford-based assay (Bio-Rad Laboratories, Inc., Hercules, CA, USA), and samples were diluted to $500 \mu \mathrm{g}$ per $2 \mathrm{ml}$ total volume with reaction buffer. Samples were pre-incubated for $30 \mathrm{~min}$ at room temperature with the specific MAO-B inhibitor, pargyline hydrochloride $(1 \mu \mathrm{M})$, and the MAO-A inhibitor, clorgyline hydrochloride $(1 \mu \mathrm{M})$. Subsequent to incubation, samples were added to individual wells of a 96-well microplate. The fluorimetric assay was initiated by adding $100 \mu \mathrm{l}$ of a reaction mixture containing Amplex Red reagent $(400 \mu \mathrm{M})$, horseradish peroxidase (HRP; $2 \mathrm{U} / \mathrm{ml}$ ) and benzylamine $(2 \mathrm{mM})$, a specific substrate of MAO-B. Plates were incubated for $30 \mathrm{~min}$ at room temperature, protected from light, and fluorescence was measured at excitation and emission wavelengths of 550 and $590 \mathrm{~nm}$, respectively, using a microplate fluorometer (Berthold Technologies, Bad Wildbad, Germany). $\mathrm{H}_{2} \mathrm{O}_{2}(10 \mu \mathrm{M})$ was used as a positive control, and reaction buffer alone was used as a negative control.

Measurement of oxygen consumption rate and extracellular acidification rate. Oxygen consumption rate (OCR) and extracellular acidification rate (ECAR) were measured using a Seahorse Bioscience XF24 Analyzer (Agilent Technologies, Inc., Santa Clara, CA, USA), a 24-well format system that automatically delivers drugs via injection ports during the assay. Prior to plating KG-1 $\alpha$ cells $\left(1 \times 10^{4}\right.$ cells/well), the XF24 sensor cartridge was initially activated by incubating with $1 \mathrm{ml}$ calibrant solution for $8 \mathrm{~h}$ at $37^{\circ} \mathrm{C}$ under normal atmospheric $\mathrm{CO}_{2}$ conditions. Following incubation for $24 \mathrm{~h}$, the cells were treated with L-deprenyl for $24 \mathrm{~h}$ or treated acutely by drug delivery via port A. Cells were then washed with XF assay media, consisting of DMEM ( $\mathrm{pH}$ 7.4) supplemented with $10 \mathrm{mM}$ glucose, $1 \mathrm{mM}$ sodium pyruvate and $2 \mathrm{mM}$ L-glutamine (without sodium bicarbonate), after which $450 \mu$ XF24 assay media was added to each well. Following equilibration for $20 \mathrm{~min}$, each well of the XF24 cartridge was sequentially injected with the ATPase inhibitor oligomycin $(2 \mu \mathrm{g} / \mathrm{ml})$, the uncoupling agent carbonyl cyanide 3-chlorophenylhydrazone $(5 \mu \mathrm{M})$ and the mitochondrial electron transport inhibitor rotenone $(2 \mu \mathrm{M})$ (all Sigma-Aldrich; Merck KGaA), and OCR and ECAR were measured in real-time.

Western blot analysis. Subsequent to the KG-1 $\alpha$ cells being washed with ice-cold $20 \mathrm{mM}$ PBS ( $\mathrm{pH} 7.4$ ), proteins were extracted using radioimmunoprecipitation assay lysis buffer containing $10 \%$ phosphatase and protease inhibitor cocktail
(Roche Diagnostics). Following centrifugation of the lysates at $14,000 \mathrm{x}$ g for $15 \mathrm{~min}$ at $4^{\circ} \mathrm{C}$, supernatants were collected and protein concentration was determined using a Bradford-based assay. Proteins in cleared lysates (10 $\mu \mathrm{g} / \mathrm{sample})$ were resolved by sodium dodecyl sulfate-polyacrylamide gel electrophoresis on $7 \%$ or $10.5 \%$ gels, and then transferred to a nitrocellulose membrane. Non-specific binding was blocked by incubating the membrane with Tris-buffered saline plus Tween-20 [10 mM Tris- $\mathrm{HCl}$ (pH 7.6), $150 \mathrm{mM} \mathrm{NaCl}$ and $0.1 \%$ Tween-20] containing 5\% skimmed milk for $1 \mathrm{~h}$. Thereafter, membranes were firstly incubated at $4^{\circ} \mathrm{C}$ overnight with the following primary antibodies: Anti-poly (ADP-ribose) polymerase 1 (PARP-1; rabbit polyclonal; 1:1,000 dilution; cat. no. sc-7150; Santa Cruz Biotechnology, Inc., Dallas, TX, USA), anti-actin (rabbit polyclonal; 1:2,000 dilution; cat. no. sc-1616; Santa Cruz Biotechnology, Inc.), anti-caspase-3 (rabbit polyclonal; 1:1,000 dilution; cat. no. 9662s; Cell Signaling Technology, Inc., Danvers, MA, USA) and anti-cleaved caspase-3 (rabbit polyclonal; 1:500 dilution; cat. no. 9661; Cell Signaling Technology, Inc.). The membranes were then incubated with the appropriate HRP-conjugated anti-immunoglobulin G secondary antibody (1:2,000 dilution; cat. no. 12-348; EMD Millipore, Billerica, MA, USA). Immunoreactive proteins were detected using an enhanced chemiluminescence (ECL) system (WEST-ZOL plus; Intron Biotechnology, Inc., Seongnam, South Korea). The quantification of band intensity was normalized with actin using Image $\mathbf{J}$ program (version 1.52c; National Institutes of Health, Bethesda, MD, USA).

ATP. Total ATP content was measured using an ATPlite assay in KG-1 $\alpha$ cells (PerkinElmer, Inc., Waltham, MA, USA) according to the manufacturer's protocols. The resulting luminescence emitted by the ATP-dependent luciferase reaction was measured using a Lumino Plate-reader (Thermo Labsystems, Santa Rosa, CA, USA).

Reverse transcription-quantitative polymerase chain reaction (RT-qPCR) analysis. Total RNA was isolated in KG-1 $\alpha$ cells using an eCube Tissue RNA Mini kit (Ep52050; PhileKorea, Seoul, South Korea) and cDNA was synthesized from $1 \mu \mathrm{g}$ total RNA using Moloney murine leukemia virus reverse transcriptase, 5X First-Strand Buffer, dithiothreitol (DTT) (cat. no. 28025-021; Invitrogen; Thermo Fisher Scientific, Inc.), deoxynucleotide triphosphates (cat. no. U15111; Promega Corporation, Madison, WI, USA) and Oligo(dT) ${ }_{15}$ primer (cat. no. C1101; Promega Corporation). RT-qPCR was performed on a 7500 Real-Time PCR system (Applied Biosystems; Thermo Fisher Scientific, Inc.) using cDNA, SYBR Green PCR Master mix (iCycleriQ Real-Time PCR Detection system; Bio-Rad Laboratories, Inc.), and the following primer pairs (designed using Primer3 version 0.4.0; Whitehead Institute, Cambridge, MA, USA and Howard Hughes Medical Institute, Chevy Chase, MD, USA): Hexokinase-1 (HK1) forward, 5'-GGC CACGATGTAGTCACCTT-3' and reverse, 5'-CACGTC CAGGTCAAATTCCT-3'; phosphofructokinase-1 (PFK1) forward, 5'-AGAGGGTTTCGATGATGCTT-3' and reverse, 5'-GTTGTAGGCAGCTCGGAGTC-3'; pyruvate dehydrogenase (lipoamide) $\alpha 1$ (PDHA) forward, 5'-AGAACTTCT ACGGGGGCAAT-3' and reverse, 5'-CGAATATCTGGC CCTGGTTA-3'; isocitrate dehydrogenase-2 (IDH2) forward, 
A

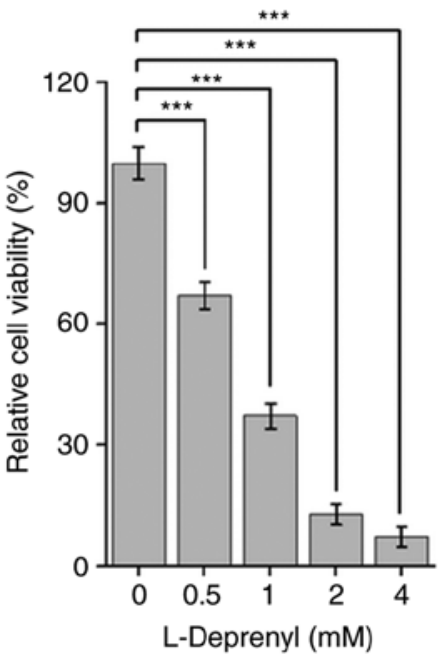

B

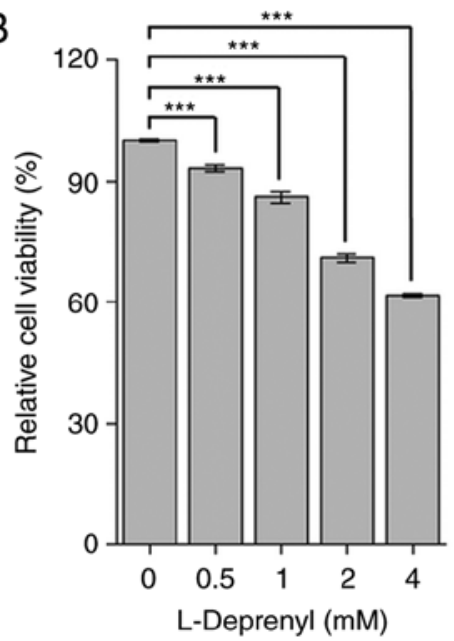

C

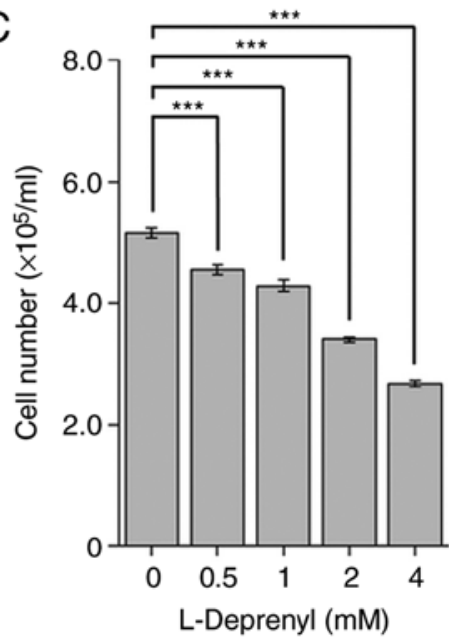

D

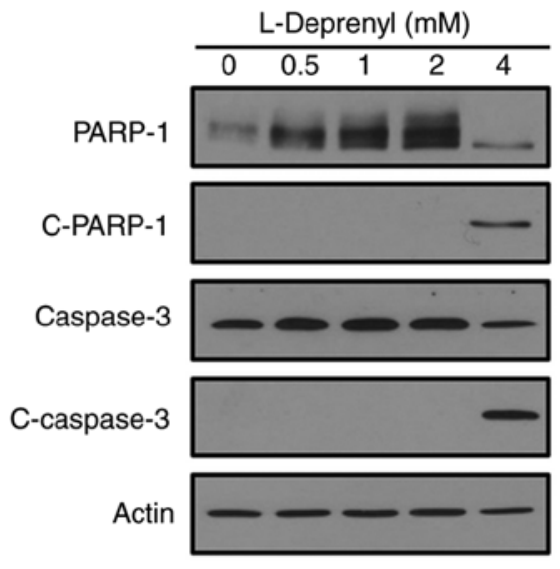

$\mathrm{E}$

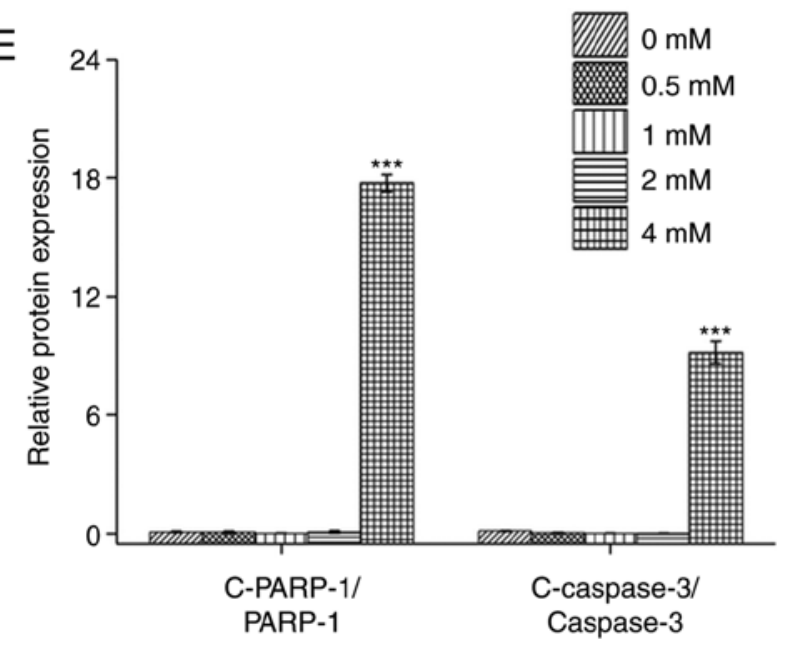

Figure 1. L-Deprenyl induces cell death in BMMNCs and KG-1 $\alpha$ cells. (A) Relative cell viability of isolated BMMNCs in FLT3-ITD knock-in mice, measured using a Cell Counting Kit-8 assay. Cells were exposed to different concentrations (0.5-4 mM) of L-deprenyl for 24 h. (B) Relative cell viability following treatment with L-deprenyl in KG-1 $\alpha$ cells. Cells were exposed to different concentrations ( $0.5-4$ mM) of L-deprenyl for 24 h. (C) Cell counts obtained using a hemocytometer following treatment of KG-1 $\alpha$ cells with different concentrations of L-deprenyl for $24 \mathrm{~h}$. (D) Western blot analysis of KG-1 $\alpha$ cells with anti-PARP-1 and anti-caspase-3 antibodies following treatment with L-deprenyl for $24 \mathrm{~h}$. (E) The densitometry analysis of panel D for ratio of cleaved form/total form, using Image J program. Data are presented as the means \pm standard error of the mean (error bars) $\left(^{* * *} \mathrm{P}<0.001 \mathrm{vs}\right.$. untreated controls). C-, cleaved; PARP-1, poly (ADP-ribose) polymerase 1; BMMNCs, bone marrow mononuclear cells.

5'-CTCATCAGGTTTGCCCAGAT-3' and reverse, 5'-GTC CGTGGTGTTCAGGAAGT-3'; NADH dehydrogenase $1 \alpha$-subcomplex subunit 9 (NDUFA9) forward, 5'-CGAGAC TGGGAAACCAAAAA-3' and reverse, 5'-GCTTCCTTG GACAGTTGAGC-3'; and 18S rRNA forward, 5'-CTGGTT GATCCTGCCAGTAG-3' and reverse, 5'-CGACCAAAG GAACCATAACT-3'. The PCR amplification process was as follows: 5 min denaturation at $95^{\circ} \mathrm{C}$, followed by 40 cycles at $95^{\circ} \mathrm{C}$ for $20 \mathrm{sec}, 60^{\circ} \mathrm{C}$ for $15 \mathrm{sec}$ and $72^{\circ} \mathrm{C}$ for $15 \mathrm{sec}$. Relative expression of target mRNAs was quantified and normalized with respect to that of $18 \mathrm{~S}$ rRNA, which was used as an endogenous control, using the $2-^{\Delta \Delta \mathrm{Cq}}$ method (14).

Statistical analysis. GraphPad Prism (GraphPad Software, Inc., San Diego, CA, USA) was used for all statistical analyses. All experiments were performed 3-4 times, and results are presented as the mean \pm standard error of the mean. The significance of differences between the groups was analyzed using one-tailed Student's t-test or one-way analysis of variance with Dunnett's multiple comparison test. $\mathrm{P}<0.05$ was considered to indicate a statistically significant difference. Individual P-values are indicated in figure legends.

\section{Results}

L-Deprenyl induces cell death in BMMNCs and KG-1 $\alpha$ cells. A previous study reported that L-deprenyl inhibits neurotoxin-induced apoptosis at low concentrations $\left(10^{-9}\right.$ to $\left.10^{-13} \mathrm{M}\right)$, but induces apoptosis at a high concentration $\left(10^{-3} \mathrm{M}\right)$ in tissues of neuro-ectodermal origin (11). Considering the focus of the present study on inducing cytotoxic effects against AML cells, high concentrations of L-deprenyl were used.

As FLT3-ITD mutation is one of the most lethal mutations in AML (15) and FLT3-ITD knock-in mice can develop myeloproliferative disease (16), cell viability tests were performed in isolated BMMNCs of FLT3-ITD knock-in and WT mice. 
Following isolation from the bone marrow, cell viability was measured in the BMMNCs using CCK-8 $24 \mathrm{~h}$ after L-deprenyl (0.5-4 $\mathrm{mM}$ ) treatment. L-Deprenyl treatment in isolated BMMNCs of FLT3-ITD knock-in mice showed decreased cell viability in a dose-dependent manner (Fig. 1A). To measure the potency of L-deprenyl on immature leukocyte cells specifically, the cell viability of isolated BMMNCs in FLT3-ITD knock-in mice and WT mice were compared. As shown in Table I, treatment with L-deprenyl at a $2 \mathrm{mM}$ concentration showed an almost two-fold potent effect on isolated BMMNCs in FLT3-ITD knock-in mice compared with that in WT mice.

To identify the type of cell death induced by L-deprenyl in leukemia cells, the cell viability was assessed in $\mathrm{KG}-1 \alpha$ cells, one of the AML cell lines. Consistent with the result in the BMMNCs, cell viability was reduced in the KG-1 $\alpha$ cells in a dose-dependent manner. The results were confirmed by CCK- 8 assay and by counting cell number using a hemocytometer (Fig. 1B and C). Moreover, an increase in cleaved PARP-1 and caspase-3, markers of apoptosis, was detected by western blotting following treatment with 4 mM L-deprenyl (Fig. 1D and E). These results suggest that prolonged treatment with L-deprenyl induces apoptotic cell death in BMMNCs and KG-1 $\alpha$ cells.

Bone marrow cells and AML cell lines lack MAO-A and B activity. Given that L-deprenyl selectively inhibits MAO-B, the present study tested for the presence of MAO-B activity in bone marrow cells and whole brain tissue, used as a positive control. As expected, whole brain possessed MAO-B activity; notably, however, bone marrow cells did not (Fig. 2A). Previous reports have indicated that cancer cells (glioma) express high levels of MAO-B (17). To determine whether bone marrow-derived cancer cells possess MAO activity, MAO-A and MAO-B activity was assessed in the leukemia KG-1 $\alpha$ and HL-60 cell lines. Unexpectedly, there was no difference found whether MAO-A and -B inhibitors were used or not, which means that neither leukemic cell line showed MAO-A or -B activity (Fig. 2B and $\mathrm{C}$ ). These observations suggest that the toxic effects of L-deprenyl on KG-1 $\alpha$ cells are independent of inhibition of MAO-B enzymatic activity.

L-Deprenyl suppresses mitochondrial respiration without affecting extracellular acidification in KG-1 $\alpha$ cells. Previous studies have shown that high-dose L-deprenyl treatment causes toxic effects on cancer cells and mammary tumors through production of ROS $(12,13,18)$. Since the main sites of ROS generation are mitochondrial oxidative phosphorylation complex I and III, the mitochondrial OCR was measured using an XF24 analyzer (19). Treatment with L-deprenyl for $24 \mathrm{~h}$ inhibited mitochondrial respiration in KG-1 $\alpha$ cells (Fig. 3A and B). As depletion of mitochondrial ATP production is abruptly compensated for by upregulation of the glycolytic pathway so as to maintain intracellular ATP content (20), the ECAR, which reflects intracellular lactate production, was also measured (Fig. 3C and D). Unexpectedly, although ECAR showed a decreasing tendency, this difference did not reach statistical significance.

Acute treatment with L-deprenyl suppresses mitochondrial respiration. L-Deprenyl is rapidly absorbed into the body and reaches its highest plasma concentration within
Table I. Cytotoxic ratio of L-deprenyl in bone marrow mononuclear cells of FLT3-ITD knock-in versus WT mice.

\begin{tabular}{lc}
\hline Concentration of L-deprenyl, $\mathrm{mM}$ & Toxicity ratio \\
\hline 0 & 1.00 \\
0.5 & 1.55 \\
1 & 1.89 \\
2 & 1.91 \\
4 & 1.75 \\
\hline
\end{tabular}

FLT3-ITD, FMS-like tyrosine kinase 3-Internal tandem duplication; WT, wild-type.
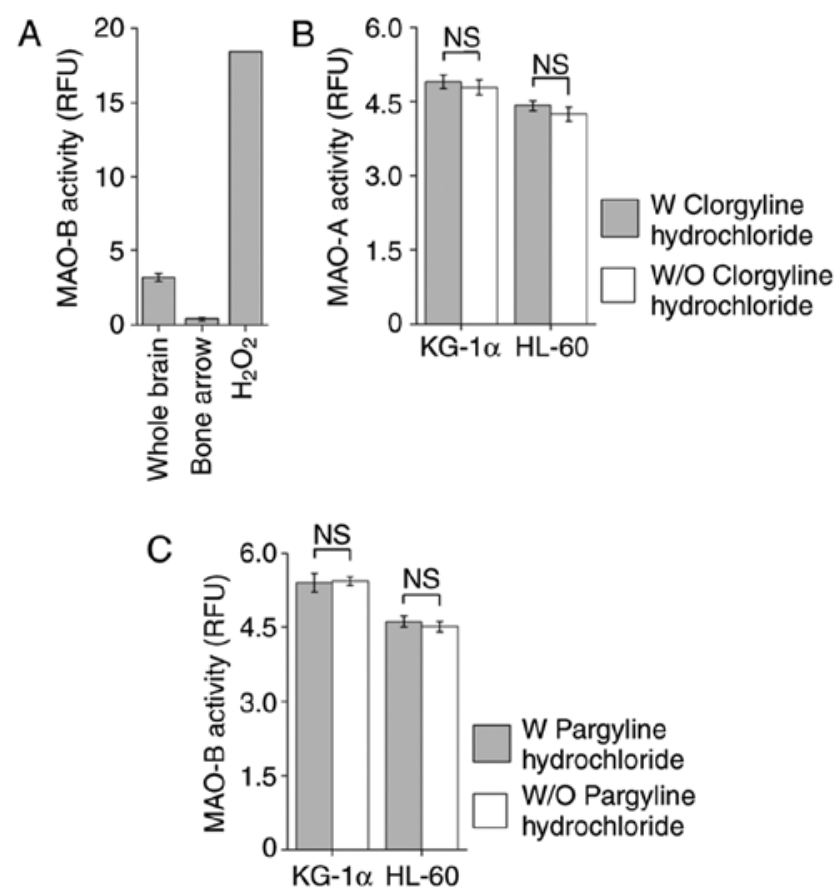

Figure 2. Lack of MAO-A and B activity in bone marrow cells and acute myeloid leukemia cell lines. (A) MAO-B activity in whole mouse brain and bone marrow cells, determined using an Amplex Red Monoamine Oxidase Assay kit. $\mathrm{H}_{2} \mathrm{O}_{2}$ was used as a positive control. (B) MAO-A activity in KG-1 $\alpha$ and HL-60 cells, incubated with the MAO-A inhibitor, clorgyline hydrochloride or PBS (control). (C) MAO-B activity in KG-1 $\alpha$ and HL-60 cells incubated with the MAO-B inhibitor, pargyline hydrochloride, or PBS (control). Data are presented as the mean \pm standard error of the mean (error bars). MAO, monoamine oxidase; RFU, relative fluorescence units; NS, not significant.

30-120 $\min$ (21). To investigate the effects of acute L-deprenyl treatment on KG-1 $\alpha$ cells, the cell viability was first assessed following treatment with L-deprenyl for $30 \mathrm{~min}$. It was found that cell viability was not changed over this time frame following treatment with 0.5 to $4 \mathrm{mM}$ L-deprenyl (Fig. 4A). Next, mitochondrial OCR was assessed using an XF24 analyzer following injection of L-deprenyl into 24-well XF cell plates. In contrast to the absence of an acute effect on viability, L-deprenyl decreased mitochondrial respiration, every $7 \mathrm{~min}$, by 21 to $32 \%$ in a concentration-dependent manner (Fig. 4B and C). In general, inhibition of mitochondrial respiration is accompanied by upregulation of the 

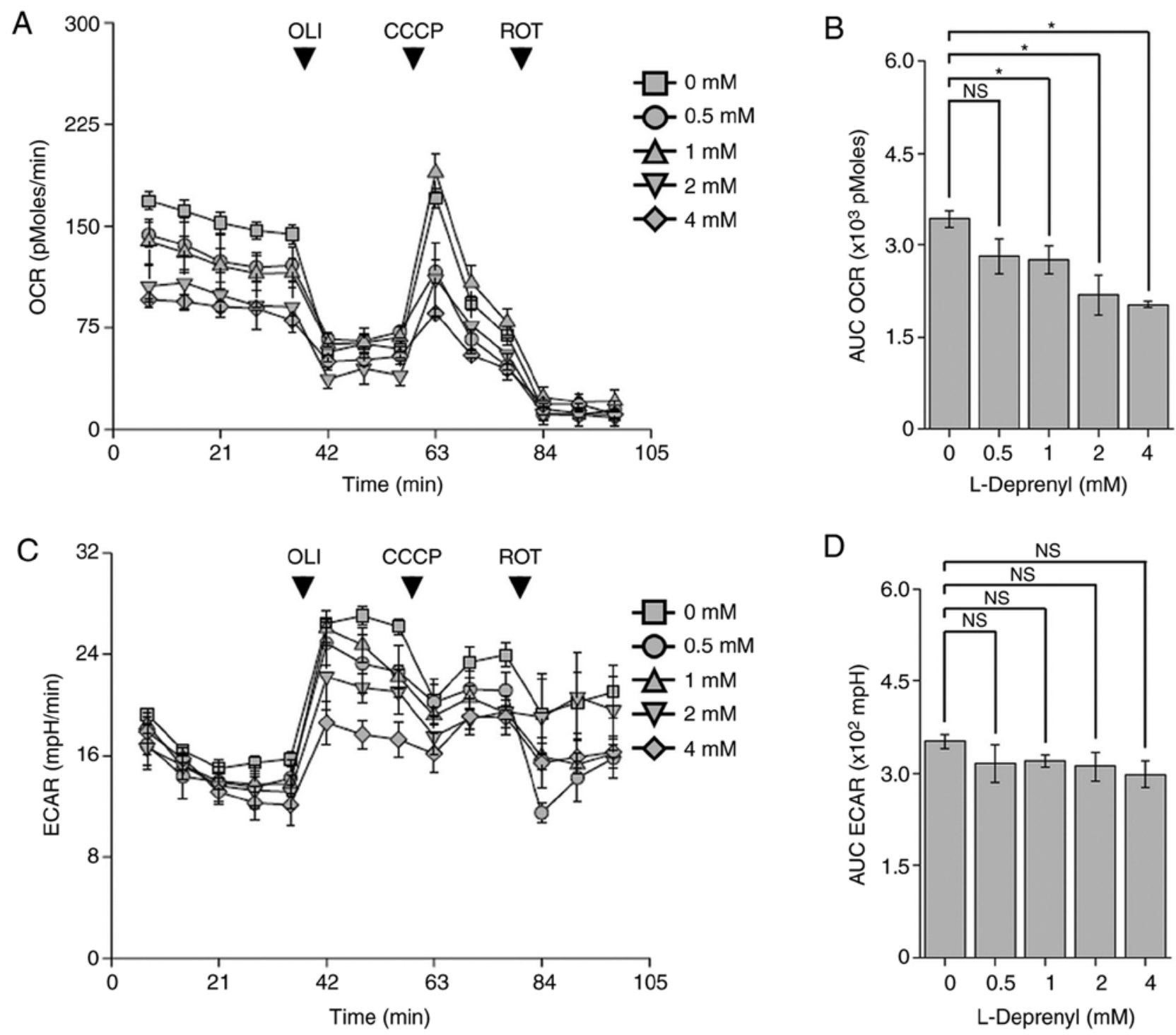

Figure 3. L-Deprenyl suppresses mitochondrial respiration without affecting glycolysis in KG-1 $\alpha$ cells. (A) OCR in KG-1 $\alpha$ cells pretreated with L-deprenyl for $24 \mathrm{~h}$. Oligomycin $(2 \mu \mathrm{g} / \mathrm{ml}), \operatorname{CCCP}(5 \mu \mathrm{M})$ and rotenone $(2 \mu \mathrm{M})$ were sequentially injected into cells. (B) The AUC of OCR, calculated using the third to fifth time points from the graphs in (A). (C) ECAR of KG-1 $\alpha$ cells after L-deprenyl pretreatment for $24 \mathrm{~h}$. Oligomycin $(2 \mu \mathrm{g} / \mathrm{ml})$, CCCP $(5 \mu \mathrm{M})$ and rotenone $(2 \mu \mathrm{M})$ were sequentially injected onto cells. (D) The AUC of ECAR, calculated using the third to fifth measurement points in graphs in (C) Data are presented as the mean \pm standard error of the mean (error bars). Each OCR and ECAR measurement point (rate) consisted of a drug injection for $2 \mathrm{~min}$, wait time for $2 \mathrm{~min}$, and detection of oxygen and hydrogen for $3 \mathrm{~min}$. "P<0.05 vs. untreated controls. OLI, oligomycin; ROT, rotenone; CCCP, carbonyl cyanide 3-chlorophenylhydrazone; OCR, oxygen consumption rate; ECAR, extracellular acidification rate; AUC, area under the curve; NS, not significant.

glycolytic pathway, which serves to maintain intracellular ATP homeostasis. As expected, L-deprenyl increased ECAR at $0.5 \mathrm{mM}$ (Fig. 4D and E); however, at higher concentrations, it decreased ECAR and led to depletion of total ATP content (Fig. 4F). Taken together with the effects of the 24-h treatment with L-deprenyl, these findings suggest that high concentration and prolonged treatment with L-deprenyl cause deterioration in the intracellular energy supply in KG-1 $\alpha$ cells and lead to apoptotic cell death.

Acute treatment with L-deprenyl alters expression levels of mRNAs for glycolysis- and tricarboxylic cycle-related genes in $K G-1 \alpha$. To investigate the physiological relevance of changes caused by acute treatment with L-deprenyl at the molecular level, changes in the expression of genes encoding proteins involved in glycolysis and the tricarboxylic acid cycle were examined. KG-1 $\alpha$ cells were treated with L-deprenyl for $20 \mathrm{~min}$, and levels of HK1, PFK1, PDHA, IDH2 and NDUFA9 mRNAs were measured by RT-qPCR. Although there was no change in mRNA levels of HK1 (Fig. 5A), consistent with the ECAR results, a high concentration $(4 \mathrm{mM})$ of L-deprenyl reduced the level of PFK1 mRNA (Fig. 5B) and a low concentration ( $0.5 \mathrm{mM})$ of L-deprenyl induced a strong increase in PDHA mRNA levels (Fig. 5C). However, no concentration of L-deprenyl treatment changed the mRNA levels of IDH2 and NDUFA9 (Fig. 5D and E). Collectively, these results indicate that, although L-deprenyl improves glycolysis at a low concentration, thereby compensating for decreased ATP content, high concentrations of L-deprenyl reduce mitochondrial respiration and glycolysis, 
A

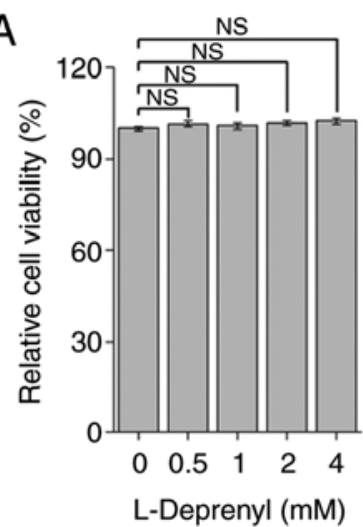

B

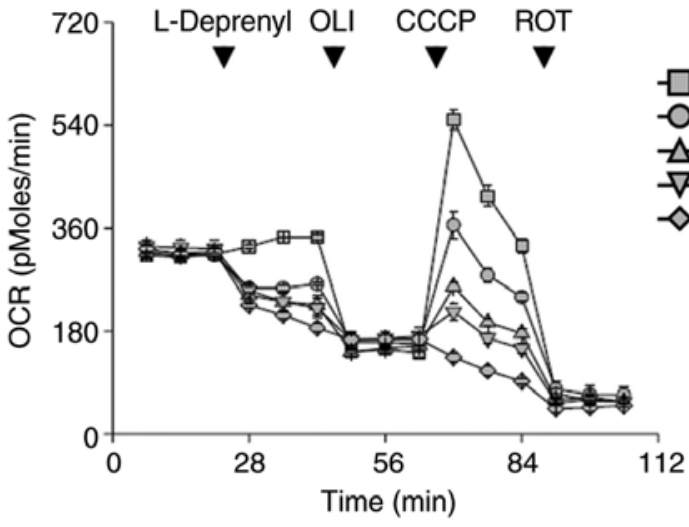

C

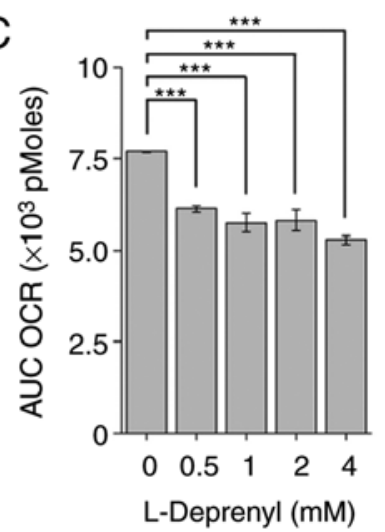

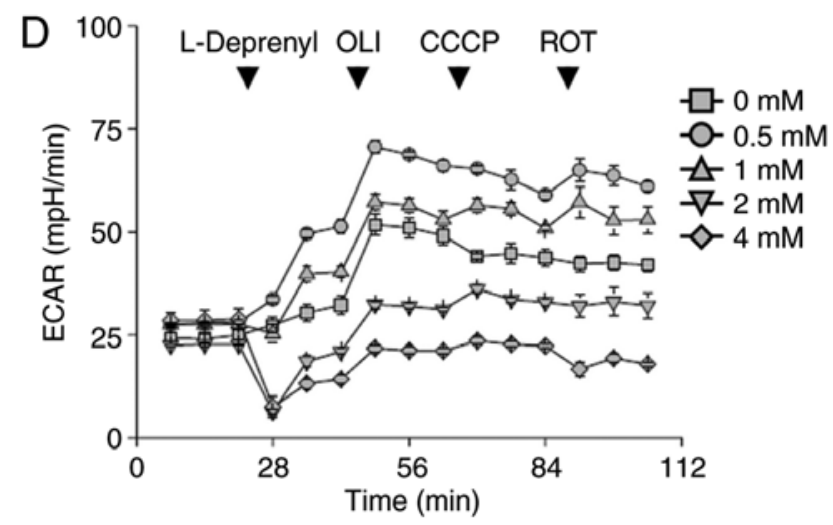
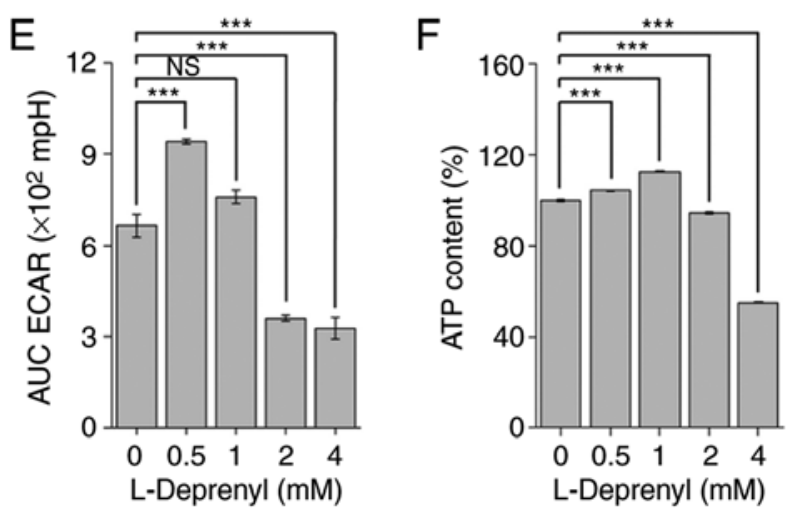

Figure 4. Acute treatment with L-deprenyl suppresses mitochondrial respiration and ATP content. (A) Viability of KG-1 $\alpha$ cells following treatment with L-deprenyl for $20 \mathrm{~min}$. (B) Real-time measurement of OCR in KG-1 $\alpha$ cells. L-Deprenyl was injected via port A of the XF24 analyzer. The four arrows indicate injection times of L-deprenyl $(0,0.5,1,2$ and $4 \mathrm{mM})$ and OCR drugs [oligomycin $(2 \mu \mathrm{g} / \mathrm{ml})$, CCCP $(5 \mu \mathrm{M})$ and rotenone $(2 \mu \mathrm{M})$ ]. (C) The AUC of OCR, calculated from the fourth to sixth time points from graphs in (A). (D) Real-time measurement of ECAR in KG-1 $\alpha$ cells. L-Deprenyl, oligomycin, CCCP and rotenone were injected in order from port A to D. Every arrow indicates injection time points of the four drugs. (E) The AUC of ECAR, calculated using the fourth to sixth measurement points in graphs in (C). (F) ATP content in KG-1 $\alpha$ cells following treatment with L-deprenyl for 20 min. Data are presented as the mean \pm standard error of the mean (error bars) $\left({ }^{* * *} \mathrm{P}<0.001\right.$ vs. untreated controls; NS, not significant). Each OCR and ECAR measurement point (rate) consisted of a drug injection for 2 min, wait time for 2 min, and detection of oxygen and hydrogen for 3 min. ATP, adenosine triphosphate; OLI, oligomycin; ROT, rotenone; CCCP, carbonyl cyanide 3-chlorophenylhydrazone; OCR, oxygen consumption rate; ECAR, extracellular acidification rate; AUC, area under the curve; NS, not significant.

and decrease ATP content, leading to cell death in the chronic phase.

\section{Discussion}

AML cells have a larger mitochondrial content than leukocytes and require mitochondrial ATP for survival and proliferation (22). The complexity of AML development involving variable genetic mutations creates challenges for achieving complete remission through genetic-targeting strategies, shifting the focus towards development of metabolic regulators. The higher content of mitochondria, the intracellular energy supply centers, in AML cells has driven the development of mitochondrial-targeting drugs. The results of the present study suggest the L-deprenyl reduces AML proliferation through inhibition of mitochondrial respiration.

Efforts to develop mitochondria-inhibiting drugs that arrest AML progression have employed molecular and genetic targeting strategies. For example, inhibition of fatty acid oxidation in the mitochondrial matrix by etomoxir and inhibition of glutaminase- 1 by bis-2-(5-phenylacetamido-1,3,4-thiadiazol-2-yl)ethyl sulfide have been studied for their potential to induce metabolic changes that suppress the energy supply required for AML proliferation $(23,24)$. In addition, AG-221 and AGI-6780 target mutant IDH2, thereby inhibiting 2-hydroxyglutarate production and inducing the differentiation of $\mathrm{AML}$ cells $(25,26)$. However, the clinical trials necessary to demonstrate the safety of these mitochondrial-targeting agents involve considerable time and expense. By contrast, L-deprenyl is already widely used to treat Parkinson's disease patients and could be rapidly enrolled as a novel therapeutic agent for AML as a mitochondrial respiration-targeting drug.

It has been reported that treatment with high-dose L-deprenyl induces toxicity against monoblastic leukemia and pituitary and mammary cancer (13,27-29). In addition, treatment of mono-mac- 6 cells with a high concentration of L-deprenyl reduces cell proliferation and metastasis (13). It has also been shown that decreasing norepinephrine and dopamine, metabolites of MAO, by treatment with L-deprenyl reduces tumor size in vivo (27-29). However, these previous reports did not identify the molecular- and organelle-based mechanism underlying the resulting apoptotic cell death. 
A

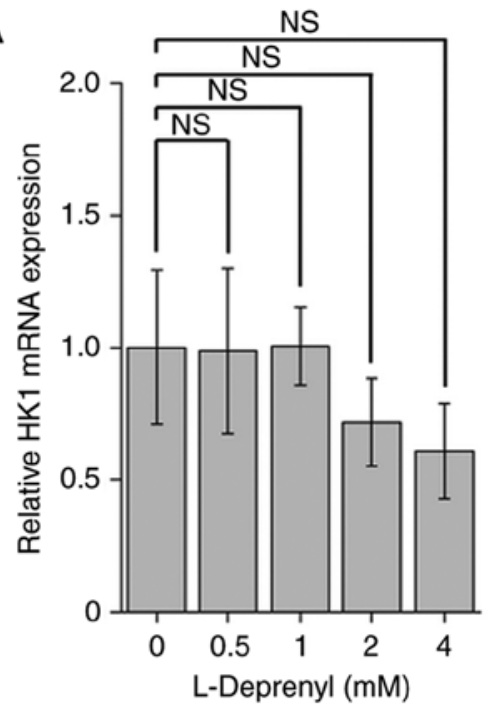

B

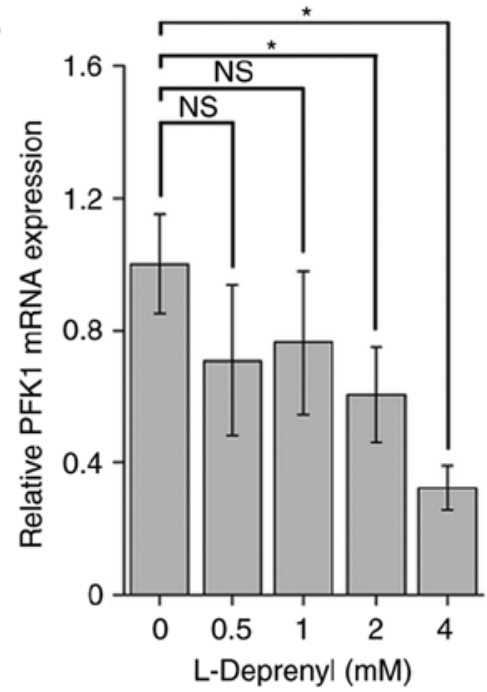

C

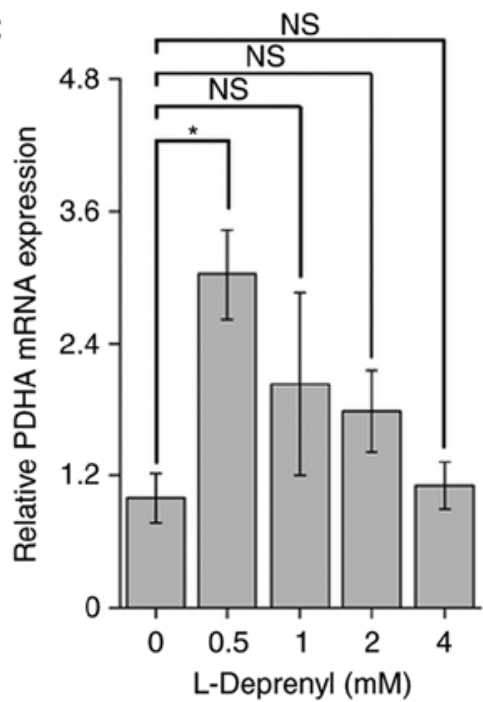

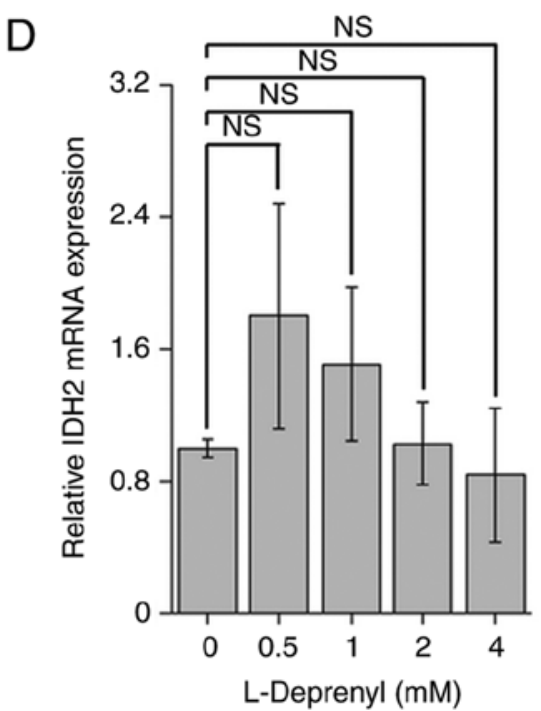

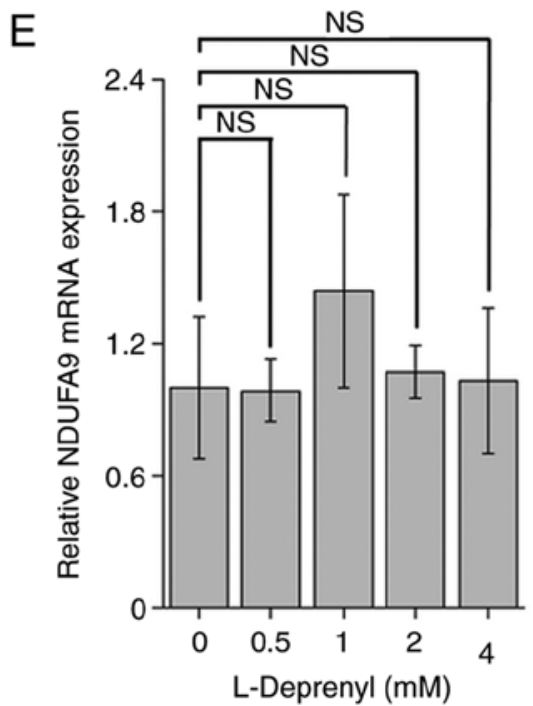

Figure 5. Expression levels of glycolysis- and TCA cycle-related genes with acute treatment of L-deprenyl in KG-1 $\alpha$. (A-E) mRNA levels of genes encoding enzymes involved in glycolysis and the TCA cycle. KG-1 $\alpha$ cells were treated with L-deprenyl for 20 min, after which (A) HK1, (B) PFK1, (C) PDHA, (D) IDH2 and (E) NDUFA9 mRNAs were quantified by reverse transcription-quantitative polymerase chain reaction. Data are presented as the mean \pm standard error of the mean (error bars) ("P<0.05 vs. untreated controls). NS, not significant; TCA, tricarboxylic acid; HK1, hexokinase-1; PFK1, phosphofructokinase-1; PDHA, pyruvate dehydrogenase (lipoamide) $\alpha 1$; IDH2, isocitrate dehydrogenase-2; NDUFA9, NADH dehydrogenase $1 \alpha$-subcomplex subunit 9.

One possible mitochondria-associated mechanism by which L-deprenyl could exert these effects would be through modulation of the stability of mitochondrial membrane potential (MMP) $(30,31)$. Low concentrations of L-deprenyl stabilize MMP in neuronal cells by increasing expression of the B-cell lymphoma 2 gene (32). As high concentrations of L-deprenyl have cytotoxic effects on the integrity of cellular homeostasis mechanisms in cancer cells, inhibition of mitochondrial respiration could be one mechanism underlying L-deprenyl-induced apoptotic cell death (13). The changes in ATP production caused by inhibition of mitochondrial respiration are consistent with the observed reduction in key glycolysis-regulating enzymes, and depletion of ATP content without compensatory glycolysis can lead to apoptotic cell death (33). Moreover, The R(-) amphetamine and $\mathrm{R}(-)$ methamphetamine produced by L-deprenyl increase the production of ROS, which impose oxidative stress on the mitochondria. As a result, mitochondria release apoptotic proteins, including cytochrome $c$ and apoptosis inducing factor, which lead to apoptotic cell death (18). Definitive confirmation that L-deprenyl-induced cell death is mitochondria-dependent will require additional studies evaluating changes in ROS in AML cells.

Trials of new organelle-targeting drugs designed to exert an anti-AML effect could pioneer a novel treatment strategy. However, the amount of time and money required for novel drug development represents an enormous hurdle. To shorten the time required to bring a drug to treat AML to the market, the clinically used drug L-deprenyl was tested in the present study and its novel targets identified. On the basis of these findings, it may be concluded that L-deprenyl causes apoptotic cell death in AML coincident with inhibition of mitochondrial OCR and cytosolic ECAR, an effect that is not mediated by MAO-B inhibition in vitro and ex vivo. In the future, regarding 
the importance of the development of a drug for controlling intracellular metabolic flux, L-deprenyl would be a candidate for first-line therapy in AML.

\section{Acknowledgements}

Not applicable.

\section{Funding}

This study was funded by National Research Foundation of Korea grants from the Ministry of Science and ICT (nos. 2016R1A2B4010398, 2016R1A6A3A11935284 and 2017R1A5A2015385) and by grants from the Ministry of Education (nos. 2014R1A6A1029617 and 2016R1D1A1B03932766) and the research fund of Chungnam National University.

\section{Availability of data and materials}

The analyzed data sets generated during the study are available from the corresponding authors on reasonable request.

\section{Authors' contributions}

IR, MJR, JYH and GRK made substantial contributions to the conception and design of the study. IR and MJR were responsible for the acquisition of data. IR, MJR and JYH assisted with the analysis and interpretation of data. IR, MJR, JYH and GRK wrote the manuscript. MJL and XJ performed the whole brain tissue preparation. BHY and YLL provided the materials for the experiments and measured the activity of MAO. JH, YJ and SJK conducted the isolation of mononuclear cells from the bone marrow. ICS, WC and EO assisted in interpreting the data and provided practical guidance. GRK and JYH are responsible for the integrity of the study as a whole. All authors read and approved the final version of the manuscript.

\section{Ethics approval and consent to participate}

All animal experiments were conducted in the animal facility according to institutional guidelines (standard operating procedure), and approved by the Institutional Animal Care and Use Committee of Chungnam National University Hospital (CNUH-015-A0007-2).

\section{Patient consent for publication}

Not applicable.

\section{Competing interests}

The authors declare that they have no competing interests

\section{References}

1. De Kouchkovsky I and Abdul-Hay M: 'Acute myeloid leukemia: A comprehensive review and 2016 update'. Blood Cancer J 6: e441, 2016

2. Löwenberg B and Rowe JM: Introduction to the review series on advances in acute myeloid leukemia (AML). Blood 127: 1, 2016.
3. Liehr T: Thorough discussion of cancer research-thoughts against the main stream. Eur J Hum Genet 25: 902, 2017.

4. Wilson CS, Davidson GS, Martin SB, Andries E, Potter J, Harvey R, Ar K, Xu Y, Kopecky KJ, Ankerst DP, et al: Gene expression profiling of adult acute myeloid leukemia identifies novel biologic clusters for risk classification and outcome prediction. Blood 108: 685-696, 2006.

5. Sallmyr A, Fan J, Datta K, Kim KT, Grosu D, Shapiro P, Small D and Rassool F: Internal tandem duplication of FLT3 (FLT3/ITD) induces increased ROS production, DNA damage, and misrepair: Implications for poor prognosis in AML. Blood 111: 3173-3182, 2008.

6. Adam-Vizi V and Chinopoulos C: Bioenergetics and the formation of mitochondrial reactive oxygen species. Trends Pharm Sci 27: 639-645, 2006.

7. Marlein CR, Zaitseva L, Piddock RE, Robinson SD, Edwards DR, Shafat MS, Zhou Z, Lawes M, Bowles KM and Rushworth SA: NADPH oxidase-2 derived superoxide drives mitochondrial transfer from bone marrow stromal cells to leukemic blasts. Blood 130: 1649-1660, 2017.

8. Watson AS, Riffelmacher T, Stranks A, Williams O, De Boer J, Cain K, MacFarlane M, McGouran J, Kessler B, Khandwala S, et al: Autophagy limits proliferation and glycolytic metabolism in acute myeloid leukemia. Cell Death Discov 1: 15008, 2015.

9. Robottom BJ: Efficacy, safety, and patient preference of monoamine oxidase B inhibitors in the treatment of Parkinson's disease. Patient Prefer Adherence 5: 57-64, 2011.

10. Youdim MB, Edmondson D and Tipton KF: The therapeutic potential of monoamine oxidase inhibitors. Nat Rev Neurosci 7: 295-309, 2006.

11. Magyar K and Szende B: (-)-Deprenyl, A selective MAO-B inhibitor, with apoptotic and anti-apoptotic properties. Neurotoxicology 25: 233-242, 2004.

12. ThyagaRajan S, Tran L, Molinaro CA, Gridley DS, Felten DL and Bellinger DL: Prevention of mammary tumor development through neuroimmunomodulation in the spleen and lymph nodes of old female sprague-dawley rats by L-Deprenyl. Neuroimmunomodulation 20: 141-151, 2013.

13. Lajkó E, Polgár L, Láng O, Lengyel J, Kőhidai L and Magyar K: Basic cell physiological activities (cell adhesion, chemotaxis and proliferation) induced by selegiline and its derivatives in Mono Mac 6 human monocytes. J Neural Transm (Vienna) 119: 545-556, 2012.

14. Livak KJ and Schmittgen TD: Analysis of relative gene expression data using real-time quantitative PCR and the 2(-Delta Delta C(T)) method. Methods 25: 402-408, 2001.

15. Chi Y, Lindgren V, Quigley S and Gaitonde S: Acute myelogenous leukemia with $\mathrm{t}(6 ; 9)(\mathrm{p} 23 ; \mathrm{q} 34)$ and marrow basophilia: An overview. Arch Pathol Lab Med 132: 1835-1837, 2008.

16. Lee BH, Tothova Z, Levine RL, Anderson K, Buza-Vidas N, Cullen DE, McDowell EP, Adelsperger J, Fröhling S, Huntly BJ, et al: FLT3 mutations confer enhanced proliferation and survival properties to multipotent progenitors in a murine model of chronic myelomonocytic leukemia. Cancer Cell 12: 367-380, 2007.

17. Sharpe MA and Baskin DS: Monoamine oxidase B levels are highly expressed in human gliomas and are correlated with the expression of HiF-1 $\alpha$ and with transcription factors $\mathrm{Sp} 1$ and $\mathrm{Sp} 3$. Oncotarget 7: 3379-3393, 2016

18. Jayanthi S, Deng X, Noailles PA, Ladenheim B and Cadet JL: Methamphetamine induces neuronal apoptosis via cross-talks between endoplasmic reticulum and mitochondria-dependent death cascades. FASEB J 18: 238-251, 2004.

19. Balaban RS, Nemoto $S$ and Finkel T: Mitochondria, oxidants, and aging. Cell 120: 483-495, 2005.

20. Pfeiffer T, Schuster S and Bonhoeffer S: Cooperation and competition in the evolution of ATP-producing pathways. Science 292: 504-507, 2001.

21. Oliver D: The structure and activity of selegiline, its functional groups, and congeners. Bioorg Chem, 2002.

22. Sriskanthadevan S, Jeyaraju DV, Chung TE, Prabha S, Xu W, Skrtic M, Jhas B, Hurren R, Gronda M, Wang X, et al: AML cells have low spare reserve capacity in their respiratory chain that renders them susceptible to oxidative metabolic stress. Blood 125: 2120-2130, 2015.

23. Samudio I, Harmancey R, Fiegl M, Kantarjian H, Konopleva M, Korchin B, Kaluarachchi K, Bornmann W, Duvvuri S, Taegtmeyer H and Andreeff M: Pharmacologic inhibition of fatty acid oxidation sensitizes human leukemia cells to apoptosis induction. J Clin Invest 120: 142-156, 2010. 
24. Jacque N, Ronchetti AM, Larrue C, Meunier G, Birsen R, Willems L, Saland E, Decroocq J, Maciel TT, Lambert M, et al: Targeting glutaminolysis has antileukemic activity in acute myeloid leukemia and synergizes with BCL-2 inhibition. Blood 126: 1346-1356, 2015.

25. Yen K, Travins J, Wang F, David MD, Artin E, Straley K, Padyana A, Gross S, DeLaBarre B, Tobin E, et al: AG-221, a first-in-class therapy targeting acute myeloid leukemia harboring oncogenic IDH2 mutations. Cancer Discov 7: 478-493, 2017.

26. Wang F, Travins J, DeLaBarre B, Penard-Lacronique V, Schalm S Hansen E, Straley K, Kernytsky A, Liu W, Gliser C, et al: Targeted inhibition of mutant IDH2 in leukemia cells induces cellular differentiation. Science 340: 622-626, 2013.

27. ThyagaRajan S, Felten SY and Felten DL: Antitumor effect of L-deprenyl in rats with carcinogen-induced mammary tumors. Cancer Lett 123: 177-183, 1998.

28. Thyagarajan S, Meites J and Quadri SK: Deprenyl reinitiates estrous cycles, reduces serum prolactin, and decreases the incidence of mammary and pituitary tumors in old acyclic rats. Endocrinology 136: 1103-1110, 1995.
29. ThyagaRajan S and Quadri SK: L-Deprenyl inhibits tumor growth, reduces serum prolactin, and suppresses brain monoamine metabolism in rats with carcinogen-induced mammary tumors. Endocrine 10: 225-232, 1999.

30. Tatton WG and Chalmers-Redman RM: Modulation of gene expression rather than monoamine oxidase inhibition: (-)-deprenyl-related compounds in controlling neurodegeneration. Neurology 47: S171-S183, 1996.

31. Simon L, Szilágyi G, Bori Z, Telek G, Magyar K and Nagy Z Low dose (-)deprenyl is cytoprotective: It maintains mitochondrial membrane potential and eliminates oxygen radicals. Life Sci 78: 225-231, 2005

32. Yi H, Maruyama W, Akao Y, Takahashi T, Iwasa K, Youdim MB and Naoi M: N-Propargylamine protects SH-SY5Y cells from apoptosis induced by an endogenous neurotoxin, $\mathrm{N}$-methyl(R) salsolinol, through stabilization of mitochondrial membrane and induction of anti-apoptotic Bcl-2. J Neural Trans 113: 21-32, 2006.

33. Liberti MV and Locasale JW: The Warburg effect: How does it benefit cancer cells? Trends Biochem Sci 41: 211-218, 2016. 